\title{
Optical measurement of azimuthal anchoring strength in nematic liquid crystals
}

\author{
Baoshe Zhang and Ping Sheng \\ Department of Physics, Hong Kong University of Science and Technology, Clear Water Bay, Kowloon, Hong Kong, China
}

Hoi S. Kwok

Center for Display Research, Hong Kong University of Science and Technology, Clear Water Bay, Kowloon, Hong Kong, China

(Received 18 September 2002; revised manuscript received 25 November 2002; published 23 April 2003)

\begin{abstract}
We have observed the azimuthal switching at the interface of nematic liquid crystals (LCs) and the aligning substrate, induced by the planar electric field of a fine comb electrode. Optical transmittance as a function of applied voltage was modeled by both the elastic theory with rigid anchoring and the Landau-de Gennes theory with the interfacial energy expression $1 / 2 W \operatorname{tr}\left[\left(\overleftrightarrow{Q}-\overleftrightarrow{Q}_{0}\right)^{2}\right]$, where $\overleftrightarrow{Q}$ is the liquid crystal order parameter and $\overleftrightarrow{Q}_{0}$ is the surface order parameter induced by the aligning substrate. Optical data on the in-plane switching LC cells were found to differ qualitatively with the predictions of the rigid anchoring model but to agree well with those of the Landau-de Gennes theory. We obtain not only the strength $W$ of the azimuthal anchoring, but also find the surface order parameter $S_{\text {surface }}$ to be 20-30\% less than that of the bulk. The optically measured azimuthal anchoring strength is in good agreement with the literature values determined through other means.
\end{abstract}

DOI: 10.1103/PhysRevE.67.041713

PACS number(s): 61.30.Gd, 78.20.Fm

In recent years, IPS (in-plane switching) techniques have been used to realize a type of liquid crystal display (LCDs) with high contrast and wide viewing angles $[1,2]$. IPS is generally achieved by using planar electric field generated by comb electrodes with a stripe width (orseparation) of $\sim 10$ $\mu \mathrm{m}$. There are two IPS mechanisms. In the rigid anchoring limit, the liquid crystal (LC) director at the substrate-LC interface is fixed, and IPS occurs through the twist of the LC director in a thin boundary layer next to the substrate. Alternatively, the interfacial anchor can be broken so that the IPS occurs partly through the reorientation of the surface director. In the latter case, the voltage dependence of optical transmittance contains information on the azimuthal surface anchoring strength. In this work, we propose to utilize IPS with narrow electrode stripes $(\sim 1 \mu \mathrm{m})$ as a microscopic probe to measure the azimuthal anchoring strength through optical means. Here the small electrode width ensures the planar electric field to be confined within a thin surface layer, hence increasing the sensitivity to the field-induced local interfacial variations. Data on optical transmittance versus voltage were found to differ qualitatively with the predictions of the rigid anchoring model but to agree well with the results of simulations assuming finite azimuthal anchoring strength.

It is well known that away from the surface layer, on the order of a few tenths of microns, LC can be accurately modeled by the elastic theory, with the LC director as the only spatially-dependent variable. Hence for the rigid anchoring model, where the director twist occurs through a length scale much larger than the surface layer, the elastic theory suffices. However, if the IPS occurs through reorientation of the surface director, then the properties of surface layer would have to be taken into account. It is known that within the surface layer there can be significant variation in the (scalar) liquid crystalline order [3-6], and the elastic theory is no longer adequate. Also, the planar electric field applied through the comb structure means that it is necessary to go beyond the usual one-dimensional (1D) model, where the electric field is assumed to be uniform and parallel to the substrate plane. In particular, in the surface layer, the LC tensorial order parameter (containing both the LC director and the scalar order parameter) is coupled to the Laplace equation through the anisotropic dielectric constant. The spatially dependent anisotropic LC dielectric constant tensor is then determined self-consistently by minimizing the total free energy. The resulting optical characteristics of the cell are calculated and compared with experiments. In the case of soft anchoring, the anchoring strength is obtained as one of the two fitting parameters.

Consider a coordinate system, where the $x-y$ plane is parallel to the substrate surface, with the $y$ axis along the electrode stripes, and $z$ axis pointing from the lower substrate towards the upper substrate. Since the length of the electrode stripes is large compared with the separation between adjacent stripes and the cell thickness, the LC cell is assumed to be uniform in the $y$ direction. Hence, the problem is $2 \mathrm{D}$ in character and periodic along the $x$ direction.

In the presence of externally applied field, the total free energy $F$ of the system may be written in the dimensionless form [7]

$$
\psi=\frac{F}{\Delta^{3} B^{4} / C^{3}}=\psi_{B}+\psi_{E},
$$

where the Landau-de Gennes bulk free energy $\psi_{B}$ and the free energy $\psi_{E}$ (resulting from the coupling between the nematic liquid crystal and the electric field) are expressed as

$$
\begin{gathered}
\psi_{B}=\int d^{3} \zeta\left\{\left(t+\frac{1}{4}\right)\left(\frac{2}{3} \bar{Q}_{i j}^{2}\right)-\frac{4}{3} \bar{Q}_{i j} \bar{Q}_{j k} \bar{Q}_{k i}+\left(\frac{2}{3} \bar{Q}_{i j}^{2}\right)^{2}\right. \\
\left.+\frac{2}{3+2 \rho} \bar{Q}_{i j, k}^{2}+\frac{2 \rho}{3+2 \rho} \bar{Q}_{i j, j} \bar{Q}_{i k, k}\right\}, \\
\psi_{E}=-\operatorname{sgn}\left(\delta \varepsilon_{a}\right) \frac{1}{3 E_{0}^{2}} \int d^{3} \zeta\left[n_{i}^{E} \bar{Q}_{i j} n_{j}^{E}\right] E^{2} .
\end{gathered}
$$


Here $\overleftrightarrow{Q}$ is the tensorial LC order parameter, $n_{i}^{E}$ denotes the $i$ th component of the unit vector along the direction of applied electric field $\vec{E}$, and $E_{0}=\sqrt{\left(4 \pi B^{3} /\left|\delta \varepsilon_{a}\right| C^{2}\right)}$ is the unit of field strength, $\delta \varepsilon_{a}=\varepsilon_{\|}-\varepsilon_{\perp}$. In Eqs. (2) and (3), the Einstein notation is assumed, with summation implied for repeated $i, j$, and $k$ indices, and comma in the subscript means derivative with respect to the spatial coordinate $(1 \rightarrow x$, $2 \rightarrow y$, and $3 \rightarrow z)$. Also $\bar{Q}_{i j}=C \overleftrightarrow{Q}_{i j} / B$ and $\vec{\zeta}=\vec{x} / \Delta(\Delta$ $\left.=\sqrt{C L / B^{2}}\right)$. For $5 \mathrm{CB}\left(4\right.$-pentyl-4'- $n^{\prime}$-cyanobiphenyl) used in the experiments described below, the Laundau-de Gennes parameter values are $a=0.065 \mathrm{~J} / \mathrm{cm}^{3} \mathrm{~K}, B=0.53 \mathrm{~J} / \mathrm{cm}^{3}$, $C=0.98 \mathrm{~J} / \mathrm{cm}^{3}, L=4.5 \times 10^{-14} \mathrm{~J} / \mathrm{cm}, \rho \cong 1[8,9]$, and $S_{\text {bulk }}$ $=0.56\left(T=20^{\circ} \mathrm{C}\right)[10]$. The perpendicular and parallel dielectric constants of $5 \mathrm{CB}$ are 5.64 and 21.3 [11], respectively. We model the interfacial potential imposed on the LC by a rubbed polyimide (PI) substrate as [12-14]

$$
\psi_{s}=\frac{1}{2} \operatorname{Wr}\left[\left(\overleftrightarrow{Q}-\overleftrightarrow{Q}_{0}\right)^{2}\right]
$$

where $W$ is the surface anchoring strength and $\overleftrightarrow{Q}_{0}$ is the tensorial interfacial order parameter. By assuming uniaxiality, $\overleftrightarrow{Q}_{0}=\frac{3}{2} S_{\text {surface }}\left(\vec{n}_{0} \vec{n}_{0}-\frac{1}{3}\right)$, where $S_{\text {surface }}$ is the surface order and $\vec{n}_{0}$ is the surface director. The surface potential imposes a penalty (for $W>0$ ) on these configurations that fail to align at the boundary with $\overleftrightarrow{Q}_{0}$. It should be noted that in general, the surface potential depends on the polar angle $\theta$ and azimuthal angle $\varphi$. Here $\theta$ is the angle between the LC director and the substrate surface, and $\varphi$ is the angle between the projection of the director on the substrate plane and the $x$ axis. The polar anchoring strength is usually much stronger than the azimuthal anchoring strength, and should be represented by a separate expression. Here we assume that the polar angle is fixed at the pretilt angle of PI. That leaves only the weaker azimuthal anchoring energy to be determined.

It should be noted that the elastic continuum theory of liquid crystals can be obtained from the Landau-de Gennes theory by treating the scalar order parameter as having no spatial variation. Hence, the free energy depends only on the director $\vec{n}$ :

$$
\begin{aligned}
F_{d}= & \frac{1}{2} \int d \vec{x}\left\{\left[k_{11}(\vec{\nabla} \cdot \vec{n})^{2}+k_{22}(\vec{n} \cdot \vec{\nabla} \times \vec{n})^{2}+k_{33}(\vec{n} \times \vec{\nabla} \times \vec{n})^{2}\right]\right. \\
& \left.-\frac{1}{2 \pi} \delta \varepsilon_{0}[\vec{E} \cdot \vec{n}]^{2}\right\}
\end{aligned}
$$

where $k_{11}, k_{22}$, and $k_{33}$ are the splay, twist, and bend Frank elastic constants, respectively.

The electrical potential $\phi$ satisfies the Laplace equation

$$
\nabla \cdot \stackrel{\leftrightarrow}{\varepsilon} \nabla \phi=0
$$

where the LC (static or low frequency) dielectric tensor $\overleftrightarrow{\varepsilon}$ $=\varepsilon_{a}+\frac{2}{3} \delta \varepsilon_{a} \overleftrightarrow{Q}$ with $\varepsilon_{0}=\left(\varepsilon_{\|}+2 \varepsilon_{\perp}\right) / 3$. The boundary condition is given by $\phi=V / 2$ and $\phi=-V / 2$ on alternate electrode stripes on the bottom substrate (Fig. 1), each $1 \mu \mathrm{m}$ in width, with center to center separation of $2 \mu \mathrm{m}$. The top substrate

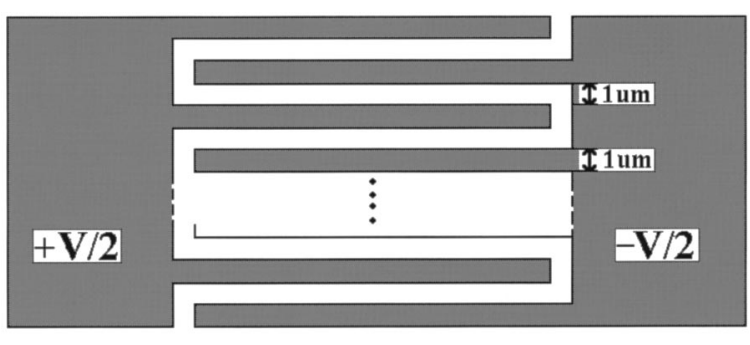

FIG. 1. A schematic diagram of the comb electrodes on an ITO glass substrate.

does not have the comb structure and is grounded. Both the top and bottom substrates are rubbed along the same (stripe) direction. Numerical simulation of IPS proceeds through a two-part iterative process whereby the solution for the electric potential $\phi$ is obtained from the Laplace Eq. (6), assuming a fixed $\overleftrightarrow{Q}$ (or $\vec{n}$ for the elastic theory) configuration, determined initially by minimizing the LC free energy at $V$ $=0$. The $\overleftrightarrow{Q}(\vec{n})$ configuration is then updated by using the conjugate gradient method, assuming a fixed potential distribution obtained previously. These two parts are repeated within each step until consistency is achieved. Optical characteristics are then calculated by using an extension of the $4 \times 4$ matrix method of Berreman [15]. This is because the original Berreman approach applies only to the propagation of polarized light in a stratified media, i.e., media that are uniform in their dielectric properties in each $x-y$ plane. Due to the comb electrodes, this assumption is no longer valid. In the Appendixes, we outline our extension of the Berreman approach. We have used the generalized matrix approach to calculate light transmittance in our 2D liquid crystal cells, using the ordinary and extraordinary refractive indices of $n_{0}=1.5074$ and $n_{e}=1.6661$ [11]. It turns out that because the $x-y$ inhomogeneities (due to the comb electrodes) exist only in a thin layer, the diffraction effect is very weak. Thus in what follows the transmittance is defined to be the zeroth order component. There is no special difference in treating the electrical potential in the case of the elastic theory, except the dielectric tensor is now expressed as $\overleftrightarrow{\varepsilon}(\vec{r})=\varepsilon_{\perp}$ $+\delta \varepsilon_{a} \vec{n}(\vec{r}) \vec{n}(\vec{r})$.

By using photolithographical techniques, we have etched comblike ITO (indium tin oxide) electrodes on one substrate (see the schematic shown in Fig. 1). The area of the comblike electrode is $5 \times 5 \mathrm{~mm}$. PI was coated onto the ITO by spin coating, and cured at $250^{\circ} \mathrm{C}$ for $1.5 \mathrm{~h}$. Typically this process resulted in $400 \AA$ thick films. These films were then rubbed using the standard rubbing machines. Cells with a thickness of $7 \mu \mathrm{m}$ were assembled with rubbing directions aligned along the electrode stripes. Two polarizers were employed at the entrance and the exit of LC cells, with the polarization direction of the entrance polarizer along the electrode stripes and that of the exit polarizer perpendicular to the electrode stripes. Thus, the initial $V=0$ state is dark. In the experiments, we used $5 \mathrm{CB}$ without any chiral dopant. Two types of polyimide (both from Merck) with different pretilt angle were used to align the LC molecules: CU-2012 with a low pretilt angle (about $1^{\circ}$ ), and SN-7321 with a high pretilt angle (about $11^{\circ}$ ).

To obtain optical transmittance $T$ as a function of applied 


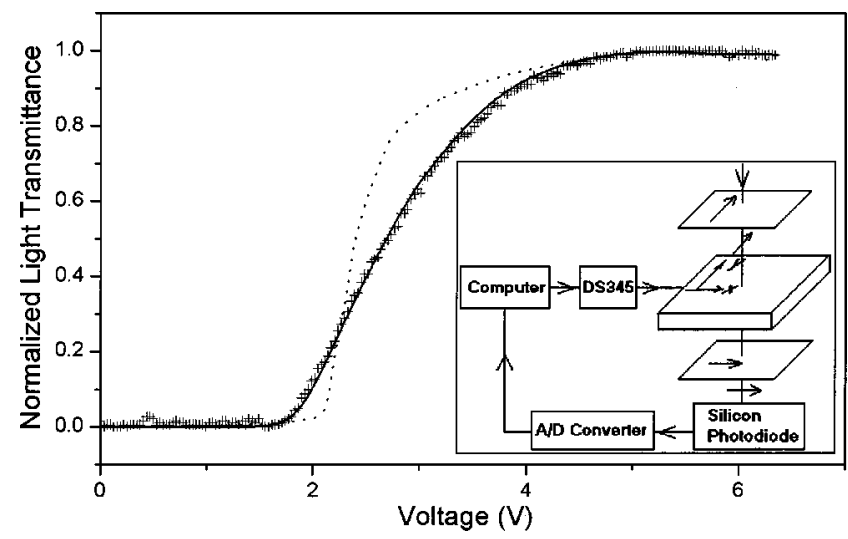

FIG. 2. Optical transmission vs the applied planar voltage for rubbed PI SN-7321. Here the symbols denote experimental data, the solid line represents the theoretical prediction with parameters given in the text, and the dash line represents the elastic theory prediction with rigid surface anchoring. The light source used is the He-Ne laser $(\lambda=632.8 \mathrm{~nm})$. The inset shows the schematic measurement arrangement.

voltage $V$, we used a He-Ne laser $(632.8 \mathrm{~nm})$ with $2 \mathrm{~mW}$ output power as the light source. The output signal was detected with a silicon photodiode. The experimental setup is shown schematically in the inset to Fig. 2. DS345 Synthesized Function Generator (Stanford Research Systems) was used as the driving voltage source. The data are shown as symbols in Figs. 2 and 3.

To explain the experimental data, we first use the rigid anchoring condition and the elastic continuum theory, calculated with $k_{11}=7.6 \times 10^{-12} \mathrm{~N}, k_{22}=5.1 \times 10^{-12} \mathrm{~N}$, and $k_{33}$ $=7.6 \times 10^{-12} \mathrm{~N}$. It was found that the predicted optical transmittance, shown as dashed lines in Figs. 2 and 3, has a relatively sharp onset as a function of voltage. This behavior is in disagreement with the experimental data but nevertheless understandable by comparison with the well-known Freedericksz transition. That is, the Fréedericksz transition represents an abrupt orientational onset when the (electric) field coherence length becomes comparable to, or smaller than, the LC cell thickness. Here the LC cell thickness is replaced by the vertical range above the substrate $\ell$ in which

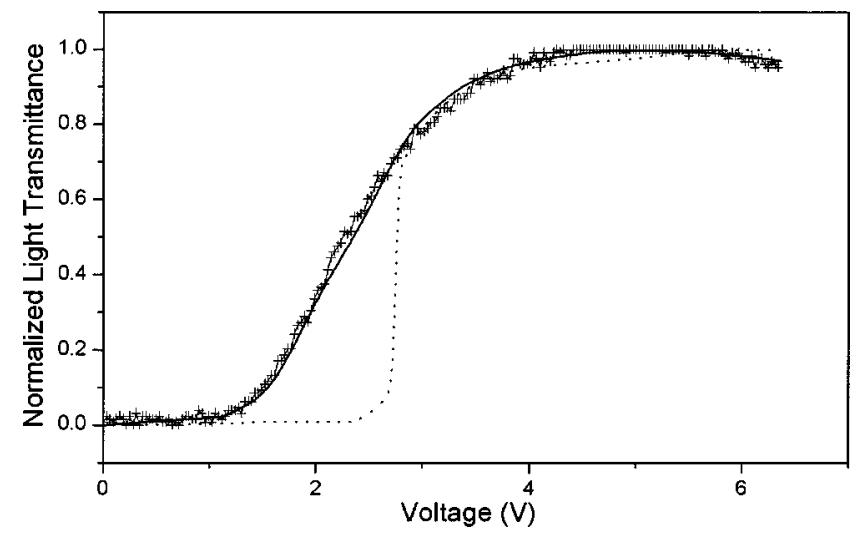

FIG. 3. Optical transmission versus the applied planar voltage for rubbed PI CU2012. The symbols and lines have the same meaning as those in Fig. 2.

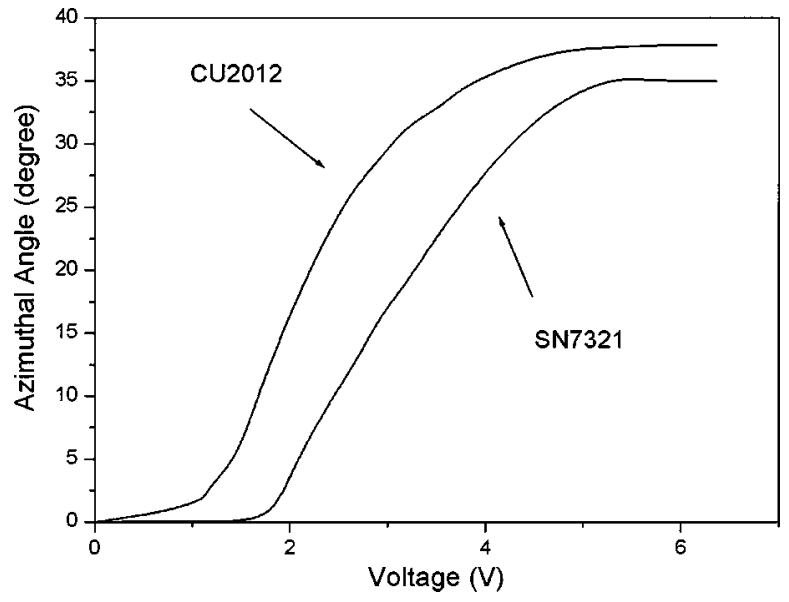

FIG. 4. Azimuthal angle variation of the LC director in the middle of the two LC cells whose transmittance is shown in Fig. 3, plotted as a function of applied voltage.

there is significant magnitude of the planar electric field. From electrostatics, $\ell$ should be on the order of periodicity along $x(2 \mu \mathrm{m})$. With rigid anchoring, the IPS is achieved through director twist over a scale defined by $\ell$. The relatively sharp onset is, therefore, a reflection of the Fréedericksz-like character. It should be noted that the sharp onset of the Fréedericksz transition is independent of the elastic constants' magnitude. Changing the elastic constants' values would only vary the onset voltage.

To simulate the IPS through the soft-anchoring approach, 20 discrete voltage values were chosen for fitting the $V-T$ experimental results. Two fitting parameters, $\eta$ $=S_{\text {surface }} / S_{\text {bulk }}$ and $K=\frac{9}{2} S_{\text {bulk }}^{2} W$, were alternately optimized to minimize mean square error between the theory and the experiment. The resulting fits are shown as solid lines in Figs. 2 and 3, with the following parameter values:

for SN-7321, $\eta=0.81$ and $K=5.22 \times 10^{-5} \mathrm{~J} / \mathrm{m}^{2}$;

for CU-2012, $\eta=0.68$ and $K=5.07 \times 10^{-5} \mathrm{~J} / \mathrm{m}^{2}$.

If there is no order parameter variation (i.e., $S_{\text {surface }}$ $=S_{\text {bulk }}$ ), Eq. (4) reduces to the standard Rapini-Papoular expression, and $K$ is the usual azimuthal anchoring strength. It is seen that the agreement between theory and experiment is excellent, with values of $K$ in the range of previously measured values of azimuthal anchoring strength (via mechanical means) obtained on rubbed polyimide surfaces [16]. These results present convincing evidence that director reorientation has indeed occurred at the LC-substrate interface, thereby providing a means of measuring the azimuthal anchoring strength through optical transmittance. In Fig. 4, we plot the attendant azimuthal angle variation of LC directors in the middle of the two LC cells.

It is also seen that there is a small difference between $K_{\mathrm{SN}-7321}$ and $K_{\mathrm{CU}-2012}$, but a significant difference between $\eta_{\mathrm{SN}-7321}$ and $\eta_{\mathrm{CU}-2012}$. In both cases, we found that $S_{\text {surface }}$ is $20 \%-30 \%$ less than $S_{\text {bulk }}$. That is, the rubbed PI substrate decreases significantly the liquid crystalline order in the interfacial layer. Thus the optical approach, coupled with IPS, 
offers a more complete picture, as well as a relatively convenient measurement method for the azimuthal anchoring strength and the related surface order.

This research was supported by CERG Grant No. HKUST6133/97P and the Hong Kong Innovation and Technology Fund.

\section{APPENDIX A}

In our 2D geometry, $\overleftrightarrow{\varepsilon}$ (dielectric constant tensor) is independent of the $y$ spatial component and periodic along the $x$ axis, i.e., $\overleftrightarrow{\varepsilon}(x+P, y, z)=\overleftrightarrow{\varepsilon}\left(x, y_{0}, z\right)$, where $P$ is the period. The incident light is a plane wave given by

$$
\begin{aligned}
& \vec{E}_{i}(x, y, z, t)=\vec{E}_{i 0} e^{-i(\omega t-\vec{k} \cdot \vec{x})}=\vec{E}_{i 0} e^{-i \omega(t-\eta x / c-\xi y / c)+i k_{z i} z}, \\
& \vec{H}_{i}(x, y, z, t)=\vec{H}_{i 0} e^{-i(\omega t-\vec{k} \cdot \vec{x})}=\vec{H}_{i 0} e^{-i \omega(t-\eta x / c-\xi y / c)+i k_{z i} z},
\end{aligned}
$$

where $\vec{E}_{i 0}$ and $\vec{H}_{i 0}$ are constant vectors and $k_{z i}$ $=(\omega / c) \sqrt{1-\eta^{2}-\xi^{2}}>0$ [without the loss of generality, we assume that the incidence plane is $z=0$ and the exit plane is $z=d(>0)]$. In Eq. (A1) $\vec{E}(x, y, z, t)$ and $\vec{H}(x, y, z, t)$ are the electromagnetic-field vectors with their respective conventional connotations. The angular frequency $\omega$ is related to the wavelength in vacuum $\lambda_{0}$, and the speed of light $c$, and the wavenumber $k_{0}$ by the relation $\omega=2 \pi c / \lambda_{0}=c k_{0}$.

From the periodicity of the dielectric constant and Eq. (A1), the electromagnetic fields can be expressed in the Bloch-wave representation:

$$
\begin{aligned}
& \vec{E}(x, y, z, t)=\vec{E}(x, z) e^{-i \omega t} e^{i k_{0}(\eta x+\xi y)}, \\
& \vec{H}(x, y, z, t)=\vec{H}(x, z) e^{-i \omega t} e^{i k_{0}(\eta x+\xi y)},
\end{aligned}
$$

where

$$
\begin{aligned}
& \vec{E}(x, z)=\vec{E}(x+P, z), \\
& \vec{H}(x, z)=\vec{H}(x+P, z) .
\end{aligned}
$$

From Maxwell equations, we obtain the following relation:

$$
\frac{\partial}{\partial z}\left(\begin{array}{c}
E_{x}(x, z) \\
H_{y}(x, z) \\
E_{y}(x, z) \\
-H_{x}(x, z)
\end{array}\right)=\left(\begin{array}{cccc}
c_{E x, E x} & c_{E x, H y} & c_{E x, E y} & -c_{E x, H x} \\
c_{H y, E x} & c_{H y, H y} & c_{H y, E y} & -c_{H y, H x} \\
c_{E y, E x} & c_{E y, H y} & c_{E y, E y} & -c_{E y, H x} \\
-c_{H x, E x} & -c_{H x, H y} & -c_{H x, E y} & c_{H x, H x}
\end{array}\right)\left(\begin{array}{c}
E_{x}(x, z) \\
H_{y}(x, z) \\
E_{y}(x, z) \\
-H_{x}(x, z)
\end{array}\right),
$$

where the matrix elements

$$
c_{A, B}=c_{A, B}\left(c, k_{0} \overleftrightarrow{\varepsilon}, \mu_{0}, \eta, \xi, \frac{\partial}{\partial x}, \frac{\partial^{2}}{\partial x^{2}}\right)\left(A, B=E_{x}, E_{y}, H_{x} \text { and } H_{y}\right)
$$

can be obtained from Eq. (B8) in Appendix B.

By utilizing a discretization scheme with $N$ points $[x=-P / 2,-(P / 2)+(P / N),-(P / 2)+2(P / N), \ldots,-(P / 2)+(N$ $-2)(P / N),-(P / 2)+(N-1)(P / N)]$ and defining

$$
\begin{aligned}
& \vec{E}_{N, x}(z)=\left(\begin{array}{c}
E_{x}(-P / 2, z) \\
E_{x}(-P / 2+P / N, z) \\
E_{x}(-P / 2+2 P / N, z) \\
\vdots \\
E_{x}(-P / 2+(N-1) P / N, z)
\end{array}\right), \quad \vec{E}_{N, y}(z)=\left(\begin{array}{c}
E_{y}(-P / 2, z) \\
E_{y}(-P / 2+P / N, z) \\
E_{y}(-P / 2+2 P / N, z) \\
\vdots \\
H_{x}(-P / 2, z) \\
H_{x}(-P / 2+P / N, z) \\
H_{x}(-P / 2+2 P / N, z) \\
\vdots \\
H_{x}(-P / 2+(N-1) P / N, z)
\end{array}\right), \quad \vec{H}_{N, y}(z)=\left(\begin{array}{c}
H_{y}(-P / 2, z) \\
H_{y}(-P / 2+P / N, z) \\
H_{y}(-P / 2+2 P / N, z) \\
\vdots \\
H_{y}(-P / 2+(N-1) P / N, z)
\end{array}\right),
\end{aligned}
$$

we obtain the following relation from Eq. (A4):

$$
\frac{\partial}{\partial z}\left(\begin{array}{c}
\vec{E}_{N, x}(z) \\
\vec{H}_{N, y}(z) \\
\vec{E}_{N, y}(z) \\
-\vec{H}_{N, x}(z)
\end{array}\right)=\left(\begin{array}{cccc}
C_{E x, E x}(z) & C_{E x, H y}(z) & C_{E x, E y}(z) & -C_{E x, H x}(z) \\
C_{H y, E x}(z) & C_{H y, H y}(z) & C_{H y, E y}(z) & -C_{H y, H x}(z) \\
C_{E y, E x}(z) & C_{E y, H y}(z) & C_{E y, E y}(z) & -C_{E y, H x}(z) \\
-C_{H x, E x}(z) & -C_{H x, H y}(z) & -C_{H x, E y}(z) & C_{H x, H x}(z)
\end{array}\right)\left(\begin{array}{c}
\vec{E}_{N, x}(z) \\
\vec{H}_{N, y}(z) \\
\vec{E}_{N, y}(z) \\
-\vec{H}_{N, x}(z)
\end{array}\right),
$$


where $C_{A, B}\left(A, B=E_{x}, E_{y}, H_{x}\right.$, and $\left.H_{y}\right)$ is the $N \times N$ coefficient matrix, obtainable from Eq. (B8) in Appendix B. Equation (A6) can be abbreviated as

$$
\frac{\partial}{\partial z} \psi(z)=\Delta(z) \psi(z)
$$

where $\psi$ denotes a $4 N$-component vector as given in Eq. (A6). If the matrix $\Delta$ is approximately independent of $z$ over some short interval $\delta z$, then the solution of this equation can be expressed by

$$
\begin{aligned}
\psi(z+\delta z) & =e^{\Delta(z) \cdot \delta z} \psi(z) \\
& =\left(1+\delta z \Delta(z)+(\delta z)^{2} \Delta^{2}(z) / 2+\cdots\right) \psi(z) .
\end{aligned}
$$

By discretizing the LC component of the cell (with thickness h) into $M$ layers, the transmittance matrix can be expressed by

$$
\begin{aligned}
& T_{\mathrm{LC}}(h) \\
& \quad=e^{\Delta(h) / M} e^{\Delta((M-1) h / M) \cdot h / M} \cdots e^{\Delta(2 h / M) \cdot h / M)} e^{\Delta(h / M) \cdot h / M} .
\end{aligned}
$$

The total transmittance matrix can be expressed by

$$
T_{\text {total }}=T_{\text {electrode_c }} T_{\mathrm{Pl}} T_{\mathrm{LC}}(h) T_{\mathrm{Pl}} T_{\text {electrode_p }},
$$

where $T_{\text {electrode_c }}, T_{\text {electrode_p }}$, and $T_{\mathrm{Pl}}$ are the constant transmittance matrices of the comb-like ITO electrode layer at the bottom of the LC cell, the layered ITO electrode layer at the top of the LC cell, and the PI layer, respectively. We define $\psi_{i}, \psi_{r}$, and $\psi_{t}$ to be the $4 N$-component vectors of the incident light, reflective light, and transmitted light of the LC cell, respectively. The transmittance matrix relates the total field vectors at the incident plane to the field vectors at the exit plane. That is,

$$
\psi_{t}=T_{\text {total }}\left(\psi_{i}+\psi_{r}\right) .
$$

From Eq. (A3), we can rewrite the vector of the electromagnetic field as follows:

$$
\begin{aligned}
& \vec{E}(x, z)=\sum_{n=0}^{N-1} \vec{e}_{n}(z) e^{i(2 \pi / P) n x}, \\
& \vec{H}(x, z)=\sum_{n=0}^{N-1} \vec{h}_{n}(z) e^{i(2 \pi / P) n x},
\end{aligned}
$$

where $(n=0, \ldots, N-1)$ ( $N$ being the number of uniformly spaced discrete points) and $[x=-P / 2,-(P / 2)+(P / N)$, $-(P / 2)+2(P / N), \ldots,-(P / 2)+(N-2)(P / N),-(P / 2)+(N$ $-1)(P / N)]$. It is noted that $e^{i(2 \pi / P) n x}(n=0, \ldots, N-1)$ constitutes a complete set of discrete orthogonal functions.

The reflected electromagnetic fields can be expressed as

$$
\begin{aligned}
\vec{E}_{r}(x, y, z, t) & =\vec{E}_{r}(x, z) e^{-i \omega t} e^{i k_{0}(\eta x+\xi y)} \\
& =e^{-i \omega t} \sum_{n=0} \vec{e}_{r, n} e^{i \vec{k}_{r, n} \cdot \vec{x}} \\
\vec{H}_{r}(x, y, z, t) & =\vec{H}_{r}(x, z) e^{-i \omega t} e^{i k_{0}(\eta x+\xi y)} \\
& =e^{-i \omega t} \sum_{n=0}^{N-1} \vec{h}_{r, n} e^{i \vec{k}_{r, n} \cdot \vec{x}}
\end{aligned}
$$

where

$$
\begin{aligned}
& \vec{E}_{r}(x, z)=\sum_{n=0}^{N-1} \vec{e}_{r, n} e^{i n(2 \pi / P) x} e^{i k_{z r, n} z}, \\
& \vec{H}_{r}(x, z)=\sum_{n=0}^{N-1} \vec{h}_{r, n} e^{i n(2 \pi / P) x} e^{i k_{z r, n} z},
\end{aligned}
$$

$\vec{e}_{r, n}=\left(e_{r, n, x}, e_{r, n, y}, e_{r, n, z}\right)$ and $\vec{h}_{r, n}=\left(h_{r, n, x}, h_{r, n, y}, h_{r, n, z}\right)$ are constant $3 \mathrm{D}$ vectors, and

$$
\vec{\kappa}_{r, n}=\vec{k}_{r, n} c / \omega=\left(\eta+n \frac{\lambda_{0}}{P}, \xi,-\sqrt{1-\left(\eta+n \frac{\lambda_{0}}{P}\right)^{2}-\xi^{2}}\right) .
$$

The transmitted electromagnetic fields can be expressed as

$$
\begin{aligned}
\vec{E}_{t}(x, y, z, t) & =\vec{E}_{t}(x, z) e^{-i \omega t} e^{i k_{0}(\eta x+\xi y)} \\
& =e^{-i \omega t} \sum_{n=0}^{N-1} \vec{e}_{t, n} e^{i \vec{k}_{t, n} \cdot\left(\vec{x}-d \hat{z}_{0}\right)}, \\
\vec{H}_{t}(x, y, z, t) & =\vec{H}_{t}(x, z) e^{-i \omega t} e^{i k_{0}(\eta x+\xi y)} \\
& =e^{-i \omega t} \sum_{n=0}^{N-1} \vec{h}_{t, n} e^{i \vec{k}_{i, n} \cdot\left(\vec{x}-d \hat{z}_{0}\right)},
\end{aligned}
$$

where $\hat{z}$ denotes the unit vector along the $z$ axis,

$$
\begin{aligned}
& \vec{E}_{t}(x, z)=\sum_{n=0}^{N-1} \vec{e}_{t, n} e^{i n(2 \pi / P) x} e^{i k_{z t, n}(z-d)}, \\
& \vec{H}_{t}(x, z)=\sum_{n=0}^{N-1} \vec{h}_{t, n} e^{i n(2 \pi / P) x} e^{i k_{z t, n}(z-d)},
\end{aligned}
$$

$\vec{e}_{t, n}=\left(e_{t, n, x}, e_{t, n, y}, e_{t, n, z}\right)$ and $\vec{h}_{t, n}=\left(h_{t, n, x}, h_{t, n, y}, h_{t, n, z}\right)$ are constant vectors, and

$$
\vec{\kappa}_{t, n}=\vec{k}_{t, n} c / \omega=\left(\eta+n \frac{\lambda_{0}}{P}, \xi, \sqrt{1-\left(\eta+n \frac{\lambda_{0}}{P}\right)^{2}-\xi^{2}}\right) .
$$

From the orthogonality of $e^{i(2 \pi / P) n x}(n=0, \ldots, N-1)$ and Maxwell equations, we have the following relations:

$$
\vec{\kappa}_{t, n} \cdot \vec{e}_{t, n}=0, \quad \vec{\kappa}_{t, n} \cdot \vec{h}_{t, n}=0,
$$




$$
\begin{gathered}
\vec{\kappa}_{t, n} \times \vec{e}_{t, n}=c \mu_{0} \vec{h}_{t, n} \quad \text { and } \quad \vec{\kappa}_{r, n} \cdot \vec{e}_{r, n}=0, \\
\vec{\kappa}_{r, n} \cdot \vec{h}_{r, n}=0, \quad \vec{\kappa}_{r, n} \times \vec{e}_{r, n}=c \mu_{0} \vec{h}_{r, n} .
\end{gathered}
$$

From Eqs. (A15) and (A19), we know that $h_{r, n, x}$ and $h_{r, n, y}$ can be represented by a linear combination of $e_{r, n, x}$ and $e_{r, n, y}$ [see Eq. (B9) in Appendix B]. Thus $\vec{H}_{N, x, r}(z=0)$ [representing the $\mathrm{N}$-dimensional vector in Eq. (A5) of the reflected magnetic field, the same notation is used below] and $\vec{H}_{N, y, r}(z=0)$ can be expressed in terms of $\vec{E}_{N, x, r}(z=0)$ and $\vec{E}_{N, y, r}(z=0)$ as

$$
\left(\begin{array}{c}
\vec{H}_{N, y}(z=0) \\
-\vec{H}_{N, x}(z=0)
\end{array}\right)_{r}=\left(\begin{array}{cc}
A_{r} & B_{r} \\
C_{r} & D_{r}
\end{array}\right)\left(\begin{array}{c}
\vec{E}_{N, x}(z=0) \\
\vec{E}_{N, y}(z=0)
\end{array}\right)_{r},
$$

where $A_{r}, B_{r}, C_{r}$, and $D_{r}$ are $N \times N$ constant matrices obtainable from Eq. (B9) in Appendix B. From Eqs. (A18) and (A19), the similar relation for the transmitted field is given by

$$
\left(\begin{array}{c}
\vec{H}_{N, y}(z=d) \\
-\vec{H}_{N, x}(z=d)
\end{array}\right)_{t}=\left(\begin{array}{cc}
A_{t} & B_{t} \\
C_{t} & D_{t}
\end{array}\right)\left(\begin{array}{l}
\vec{E}_{N, x}(z=d) \\
\vec{E}_{N, y}(z=d)
\end{array}\right)_{t},
$$

where $A_{t}, B_{t}, C_{t}$, and $D_{t}$ are $N \times N$ constant matrices. [see Eq. (B9) in Appendix B]. Therefore, $\psi_{r}$ and $\psi_{t}$ can be expressed, respectively, by

$$
\begin{aligned}
\psi_{r}= & \left(\begin{array}{c}
\vec{E}_{N, x}(z=0) \\
\vec{H}_{N, y}(z=0) \\
\vec{E}_{N, y}(z=0) \\
-\vec{H}_{N, x}(z=0)
\end{array}\right)_{r}=\left(\begin{array}{cccc}
I & 0 & 0 & 0 \\
A_{r} & B_{r} & 0 & 0 \\
0 & I & 0 & 0 \\
C_{r} & D_{r} & 0 & 0
\end{array}\right) \\
& \times\left(\begin{array}{c}
\vec{E}_{N, x, r}(z=0) \\
\vec{E}_{N, y, r}(z=0) \\
\vec{E}_{N, x, t}(z=d) \\
\vec{E}_{N, y, t}(z=d)
\end{array}\right), \\
\psi_{t}= & \left(\begin{array}{c}
\vec{E}_{N, x}(z=d) \\
\vec{H}_{N, y}(z=d) \\
\vec{E}_{N, y}(z=d) \\
-\vec{H}_{N, x}(z=d)
\end{array}\right),=\left(\begin{array}{cccc}
0 & 0 & I & 0 \\
0 & 0 & A_{t} & B_{t} \\
0 & 0 & 0 & I \\
0 & 0 & C_{t} & D_{t}
\end{array}\right) \\
& \times\left(\begin{array}{l}
\vec{E}_{N, x, r}(z=0) \\
\vec{E}_{N, y, r}(z=0) \\
\vec{E}_{N, x, t}(z=d) \\
\vec{E}_{N, y, t}(z=d)
\end{array}\right) .
\end{aligned}
$$

Combining Eqs. (A11) and (A20), we obtain the reflected fields at the incidence plane and the transmitted fields at the exit plane.

It is noted that the present approach can be simply extended to the 3D case with

$$
\left(\begin{array}{l}
\overleftrightarrow{\varepsilon}(x, y, z) \\
\overleftrightarrow{\mu}(x, y, z)
\end{array}\right)=\left(\begin{array}{l}
\overleftrightarrow{\varepsilon}\left(x+P_{x}, y, z\right) \\
\overleftrightarrow{\mu}\left(x+P_{x}, y, z\right)
\end{array}\right)
$$

and

$$
\left(\begin{array}{l}
\overleftrightarrow{\varepsilon}(x, y, z) \\
\overleftrightarrow{\mu}(x, y, z)
\end{array}\right)=\left(\begin{array}{l}
\overleftrightarrow{\varepsilon}\left(x, y+P_{y}, z\right) \\
\overleftrightarrow{\mu}\left(x, y+P_{y}, z\right)
\end{array}\right)
$$

( $\overleftrightarrow{\varepsilon}$ and $\overleftrightarrow{\mu}$ being the dielectric constant tensor and permittivity tensor respectively, $P_{x}$ and $P_{y}$ being the periodicities along the $x$ and $y$ directions, respectively).

\section{APPENDIX B}

Here we derive the relevant matrix elements used in Appendix A from the Maxwell equations. The matrix representation of the Maxwell equations is given by

$$
\left.\begin{array}{|cccccc}
0 & 0 & 0 & 0 & -\frac{\partial}{\partial z} & \frac{\partial}{\partial y} \\
0 & 0 & 0 & \frac{\partial}{\partial z} & 0 & -\frac{\partial}{\partial x} \\
0 & 0 & 0 & -\frac{\partial}{\partial y} & \frac{\partial}{\partial x} & 0 \\
0 & \frac{\partial}{\partial z} & -\frac{\partial}{\partial y} & 0 & 0 & 0 \\
-\frac{\partial}{\partial z} & 0 & \frac{\partial}{\partial x} & 0 & 0 & 0 \\
\frac{\partial}{\partial y} & -\frac{\partial}{\partial x} & 0 & 0 & 0 & 0
\end{array} \mid \begin{array}{c}
E_{x} \\
E_{y} \\
E_{z} \\
H_{x} \\
H_{y} \\
H_{z}
\end{array}\right)
$$

The constitutive equations can be written as

$$
\vec{D}=\overleftrightarrow{\varepsilon} \vec{E}=\varepsilon_{0}\left(\begin{array}{ccc}
\varepsilon_{11} & \varepsilon_{12} & \varepsilon_{13} \\
\varepsilon_{21} & \varepsilon_{22} & \varepsilon_{23} \\
\varepsilon_{31} & \varepsilon_{32} & \varepsilon_{33}
\end{array}\right)\left(\begin{array}{c}
E_{x} \\
E_{y} \\
E_{z}
\end{array}\right)
$$

and

$$
\vec{B}=\overleftrightarrow{\mu} \vec{H}=\mu_{0}\left(\begin{array}{ccc}
1 & 0 & 0 \\
0 & 1 & 0 \\
0 & 0 & 1
\end{array}\right)\left(\begin{array}{l}
H_{x} \\
H_{y} \\
H_{z}
\end{array}\right)
$$

where $\vec{E}(x, y, z, t), \vec{D}(x, y, z, t), \vec{H}(x, y, z, t)$, and $\vec{B}(x, y, z, t)$ are defined in Appendix A, and $\varepsilon_{0}$ and $\mu_{0}$ are dielectric constant and permittivity of the vacuum, respectively. By 
combining Eqs. (B1)-(B3) and assuming harmonic time variation [i.e., $\vec{E}, \vec{H}(x, y, z, t)=\vec{E}$, and $\vec{H}(x, y, z) e^{-i \omega t}$ ], the Maxwell equations become

$$
\begin{array}{|cccccc}
0 & 0 & 0 & 0 & -\frac{\partial}{\partial z} & \frac{\partial}{\partial y} \\
0 & 0 & 0 & \frac{\partial}{\partial z} & 0 & -\frac{\partial}{\partial x} \\
0 & 0 & 0 & -\frac{\partial}{\partial y} & \frac{\partial}{\partial x} & 0 \\
0 & \frac{\partial}{\partial z} & -\frac{\partial}{\partial y} & 0 & 0 & 0 \\
-\frac{\partial}{\partial z} & 0 & \frac{\partial}{\partial x} & 0 & 0 & 0 \\
\frac{\partial}{\partial y} & -\frac{\partial}{\partial x} & 0 & 0 & 0 & 0
\end{array} \mid\left(\begin{array}{c}
E_{x} \\
E_{y} \\
E_{z} \\
c \mu_{0} H_{x} \\
c \mu_{0} H_{y} \\
c \mu_{0} H_{z}
\end{array}\right)
$$

where

$$
M=\left(\begin{array}{cccccc}
\varepsilon_{11} & \varepsilon_{12} & \varepsilon_{13} & 0 & 0 & 0 \\
\varepsilon_{21} & \varepsilon_{22} & \varepsilon_{23} & 0 & 0 & 0 \\
\varepsilon_{31} & \varepsilon_{32} & \varepsilon_{33} & 0 & 0 & 0 \\
0 & 0 & 0 & 1 & 0 & 0 \\
0 & 0 & 0 & 0 & 1 & 0 \\
0 & 0 & 0 & 0 & 0 & 1
\end{array}\right) .
$$

If the electric and magnetic fields can be represented in the form

$$
\begin{aligned}
& \vec{E}(x, y, z)=\vec{E}(x, z) e^{i k_{0}(\eta x+\xi y)}, \\
& \vec{H}(x, y, z)=\vec{H}(x, z) e^{i k_{0}(\eta x+\xi y)},
\end{aligned}
$$

where $\eta$ and $\xi$ are constants, then the Maxwell equations in the matrix form can be further simplified as follows:

$$
\begin{aligned}
& \left.\begin{array}{|cccccc}
0 & 0 & 0 & 0 & -\frac{\partial}{\partial\left(k_{0} z\right)} & i \xi \\
0 & 0 & 0 & \frac{\partial}{\partial\left(k_{0} z\right)} & 0 & -i \eta-\frac{\partial}{\partial\left(k_{0} x\right)} \\
0 & \frac{\partial}{\partial\left(k_{0} z\right)} & -i \xi & 0 & 0 & 0 \\
-\frac{\partial}{\partial\left(k_{0} z\right)} & 0 & i \eta+\frac{\partial}{\partial\left(k_{0} x\right)} & 0 & 0 & 0 \\
i \xi & -i \eta-\frac{\partial}{\partial\left(k_{0} x\right)} & 0 & 0 & 0 & 0
\end{array} \mid \begin{array}{c}
E_{x}(x, z) \\
E_{y}(x, z) \\
E_{z}(x, z) \\
c \mu_{0} H_{x}(x, z) \\
c \mu_{0} H_{y}(x, z) \\
c \mu_{0} H_{z}(x, z)
\end{array}\right) \\
& =-i M\left(\begin{array}{c}
E_{x}(x, z) \\
E_{y}(x, z) \\
E_{z}(x, z) \\
c \mu_{0} H_{x}(x, z) \\
c \mu_{0} H_{y}(x, z) \\
c \mu_{0} H_{z}(x, z)
\end{array}\right) \text {. }
\end{aligned}
$$


From Eq. (B6) we obtain

$$
\begin{gathered}
E_{z}=\frac{1}{\varepsilon_{33}}\left[\xi\left(c \mu_{0} H_{x}\right)-\eta\left(c \mu_{0} H_{y}\right)+i \frac{\partial}{\partial\left(k_{0} x\right)}\left(c \mu_{0} H_{y}\right)\right] \\
-\frac{1}{\varepsilon_{33}}\left(\varepsilon_{31} E_{x}+\varepsilon_{32} E_{y}\right), \\
\left(c \mu_{0} H_{z}\right)=-\xi E_{x}+\eta E_{y}-i \frac{\partial}{\partial\left(k_{0} x\right)} E_{y} .
\end{gathered}
$$

Substituting the above formula into the Maxwell equations (B6), we obtain

$$
\begin{aligned}
& \frac{\partial}{\partial\left(k_{0} z\right)} E_{x}=-\left[\frac{\partial}{\partial\left(k_{0} x\right)} \frac{\varepsilon_{31}}{\varepsilon_{33}}+i \eta \frac{\varepsilon_{31}}{\varepsilon_{33}}\right] E_{x}-\frac{\varepsilon_{31}}{\varepsilon_{33}} \frac{\partial}{\partial\left(k_{0} x\right)} E_{x} \\
&-\left[\frac{\partial}{\partial\left(k_{0} x\right)} \frac{\varepsilon_{32}}{\varepsilon_{33}}+i \eta \frac{\varepsilon_{32}}{\varepsilon_{33}}\right] E_{y}-\frac{\varepsilon_{32}}{\varepsilon_{33}} \frac{\partial}{\partial\left(k_{0} x\right)} E_{y} \\
&+\xi\left[\frac{\partial}{\partial\left(k_{0} x\right)} \frac{1}{\varepsilon_{33}}+i \eta \frac{1}{\varepsilon_{33}}\right]\left(c \mu_{0} H_{x}\right) \\
&+\xi \frac{1}{\varepsilon_{33}} \frac{\partial}{\partial\left(k_{0} x\right)}\left(c \mu_{0} H_{x}\right)+i\left[1-\eta^{2} \frac{1}{\varepsilon_{33}}\right. \\
&\left.+i \eta \frac{\partial}{\partial\left(k_{0} x\right)} \frac{1}{\varepsilon_{33}}\right]\left(c \mu_{0} H_{y}\right)+\left[i \frac{\partial}{\partial\left(k_{0} x\right)} \frac{1}{\varepsilon_{33}}\right. \\
&\left.-2 \eta \frac{1}{\varepsilon_{33}}\right] \frac{\partial}{\partial\left(k_{0} x\right)}\left(c \mu_{0} H_{y}\right) \\
&+i \frac{1}{\varepsilon_{33}} \frac{\partial^{2}}{\partial\left(k_{0} x\right)^{2}}\left(c \mu_{0} H_{y}\right), \\
&-i \frac{\eta \xi}{\varepsilon_{33}}\left(c \mu_{0} H_{y}\right)-\frac{\xi}{\varepsilon_{33}} \frac{\partial}{\partial\left(k_{0} x\right)}\left(c \mu_{0} H_{y}\right) \\
& \frac{\varepsilon_{31}}{\varepsilon_{33}} E_{x}-i \xi \frac{\varepsilon_{32}}{\varepsilon_{33}} E_{y}+i\left(\frac{\xi^{2}}{\varepsilon_{33}}-1\right)\left(c \mu_{0} H_{x}\right) \\
& E_{y}=-i \xi 8) \\
& \\
&\left.\varepsilon_{0}\right)(\mathrm{B} 8) \\
& \\
&
\end{aligned}
$$$$
\frac{\partial}{\partial\left(k_{0} z\right)}\left(c \mu_{0} H_{x}\right)=i\left[-\eta \xi-\varepsilon_{21}+\frac{\varepsilon_{23}}{\varepsilon_{33}} \varepsilon_{31}\right] E_{x}-\xi \frac{\partial}{\partial\left(k_{0} x\right)} E_{x}
$$$$
+i\left[\eta^{2}-\varepsilon_{22}+\frac{\varepsilon_{23}}{\varepsilon_{33}} \varepsilon_{32}\right] E_{y}+2 \eta \frac{\partial}{\partial\left(k_{0} x\right)} E_{y}
$$$$
-i \frac{\partial^{2}}{\partial\left(k_{0} x\right)^{2}} E_{y}-i \xi \frac{\varepsilon_{23}}{\varepsilon_{33}}\left(c \mu_{0} H_{x}\right)
$$

$$
+i \eta \frac{\varepsilon_{23}}{\varepsilon_{33}}\left(c \mu_{0} H_{y}\right)+\frac{\varepsilon_{23}}{\varepsilon_{33}} \frac{\partial}{\partial\left(k_{0} x\right)}\left(c \mu_{0} H_{y}\right),
$$

$$
\begin{aligned}
\frac{\partial}{\partial\left(k_{0} z\right)}\left(c \mu_{0} H_{y}\right)= & -i\left[\xi^{2}-\varepsilon_{11}+\frac{\varepsilon_{13}}{\varepsilon_{33}} \varepsilon_{31}\right] E_{x} \\
& +i\left[\eta \xi+\varepsilon_{12}-\frac{\partial_{13}}{\varepsilon_{33}} \varepsilon_{32}\right] E_{y}+\xi \frac{\partial}{\partial\left(k_{0} x\right)} E_{y} \\
& +i \xi \frac{\varepsilon_{13}}{\varepsilon_{33}}\left(c \mu_{0} H_{x}\right)-i \eta \frac{\varepsilon_{13}}{\varepsilon_{33}}\left(c \mu_{0} H_{y}\right) \\
& -\frac{\varepsilon_{13}}{\varepsilon_{33}} \frac{\partial}{\partial\left(k_{0} x\right)}\left(c \mu_{0} H_{y}\right) .
\end{aligned}
$$

Also, from Eqs. (A15), (A18), and (A19) in Appendix A, we have the following linear relations between $\vec{e}_{s, n}$ and $\vec{h}_{s, n}(s$ $=t, r)$ :

$$
\begin{aligned}
& c \mu_{0} h_{t, n, x}=-\frac{\xi\left(\eta+n \frac{\lambda_{0}}{P}\right) e_{t, n, x}+\left[1-\left(\eta+n \frac{\lambda_{0}}{P}\right)^{2}\right] e_{t, n, y}}{\sqrt{1-\left(\eta+n \frac{\lambda_{0}}{P}\right)^{2}-\xi^{2}}}, \\
& \frac{\left(1-\xi^{2}\right) e_{t, n, x}+\xi\left(\eta+n \frac{\lambda_{0}}{P}\right) e_{t, n, y}}{\sqrt{1-\left(\eta+n \frac{\lambda_{0}}{P}\right)^{2}-\xi^{2}}}, \\
& c \mu_{0} h_{r, n, x}=\frac{\xi\left(\eta+n \frac{\lambda_{0}}{P}\right) e_{r, n, x}+\left[1-\left(\eta+n \frac{\lambda_{0}}{P}\right)^{2}\right] e_{r, n, y}}{\sqrt{1-\left(\eta+n \frac{\lambda_{0}}{P}\right)^{2}-\xi^{2}}},
\end{aligned}
$$

$$
c \mu_{0} r_{r, n, y}=\frac{\left(1-\xi^{2}\right) e_{r, n, x}+\xi\left(\eta+n \frac{\lambda_{0}}{P}\right) e_{r, n, y}}{\sqrt{1-\left(\eta+n \frac{\lambda_{0}}{P}\right)^{2}-\xi^{2}}} .
$$

[1] M. Oh-e, M. Ohta, S. Aratani, and K. Kondo, in Proceedings of the 15th International Display Research Conference (Society for Information Display and the Institute of Television Engineers of Japan, Hamamatsu, 1995), p. 577.

[2] K. Kondo, S. Matsuyama, N. Konishi, and H. Kawakami,
1998, SID Digest 1998, p. 389.

[3] P. Sheng, Phys. Rev. Lett. 37, 1059 (1976).

[4] P. Sheng, Phys. Rev. A 26, 1610 (1982).

[5] W. Chen, M. B. Feller, and Y. R. Shen, Phys. Rev. Lett. 63, 2665 (1989); M. B. Feller, W. Chen, and Y. R. Shen, Phys. 
Rev. A 43, 6778 (1991).

[6] X. Zhuang, L. Marrucci, and Y. R. Shen, Phys. Rev. Lett. 73, 1513 (1994).

[7] T. Z. Qian and P. Sheng, Phys. Rev. Lett. 77, 4564 (1996).

[8] P. Sheng and E. Priestly, in Introduction of Liquid Crystals, edited by E. B. Priestly, P. J. Wojtowicz, and P. Sheng (Plenum Press, New York, 1975), p. 153.

[9] T. J. Sluckin and A. Poniewierski, Phys. Rev. Lett. 55, 2907 (1985); P. Sheng, B. Z. Li, M. Y. Zhou, T. Moses, and Y. R. Shen, Phys. Rev. A 46, 946 (1992); Y. L'vov, R. M. Hornreich, and D. W. Allender, Phys. Rev. E 48, 1115 (1993); N. Kothekar, D. W. Allender, and R. M. Hornreich, ibid. 49, 2150
(1994).

[10] H. J. Coles, in The Optics of Thermotropic Liquid Crystals, edited by Steve Elston and Roy Sambles (Taylor, London, 1998), pp. 57-84.

[11] 1997 Newsletter, Division of Liquid Crystals, Merck Co. (Germany).

[12] M. Nobili and G. Durand, Phys. Rev. A 46, R6174 (1992).

[13] A. di Garbo and M. Nobili, Liq. Cryst. 19, 269 (1995).

[14] T. A. Davis and E. C. Gartland, JR, SIAM (Soc. Ind. Appl. Math.) J. Numer. Anal. 35, 336 (1998).

[15] D. W. Berreman, J. Opt. Soc. Am. 62, 502 (1972).

[16] S.-H. Paek, C. J. Durning, K.-W. Lee, and A. Lien, Appl. Phys. 83, 1270 (1998). 